\title{
Microstructures using RF sputtered PSG film as a sacrificial layer in surface micromachining
}

\author{
VIVEKANAND BHATT ${ }^{1, *}$, SUDHIR CHANDRA $^{1}$ and \\ CHATAR SINGH ${ }^{2}$ \\ ${ }^{1}$ Centre for Applied Research in Electronics (CARE), \\ ${ }^{2}$ Textile Technology, Indian Institute of Technology Delhi, New Delhi 110016 \\ e-mail: vivekanand_iitd@yahoo.com
}

\begin{abstract}
In this paper, we explore RF magnetron sputtered Phosphor-silicateglass (PSG) film as a sacrificial layer in surface micromachining technology. For this purpose, a $76 \mathrm{~mm}$ diameter target of phosphorus-doped silicon dioxide was prepared by conventional solid-state reaction route using $\mathrm{P}_{2} \mathrm{O}_{5}$ and $\mathrm{SiO}_{2}$ powders. The PSG films were prepared in a RF $(13.56 \mathrm{MHz})$ magnetron sputtering system at 300 watt RF power, 20 mTorr pressure and $45 \mathrm{~mm}$ target-to-substrate spacing without external substrate heating. Microstructures of sputtered silicon dioxide film were fabricated using sputtered PSG film as sacrificial layer in surface micromachining process.
\end{abstract}

Keywords. RF sputtering; sputtered PSG film; microstructures; surface micromachining.

\section{Introduction}

Phospho-silicate-glass (PSG) films have a variety of applications in the fabrication of microelectronic devices and Microelectromechanical System (MEMS). In microelectronic industry, the PSG films have been used in the fabrication of bipolar and metal-oxide-semiconductor (MOS) devices as interlevel and intermetal dielectric films for multilevel interconnections (Kern \& Schnable 1982; Sze 1988). These films are also used for surface passivation and improving the metal layer step coverage in device fabrication (Sze 1988; Takamatsu et al 1984). In MEMS, PSG films have been reported to be one of the most suitable materials for sacrificial layer because of its high etch rate. Atmospheric Pressure Chemical Vapor Deposition (APCVD) (Kern \& Schnable 1982), Low Pressure Chemical Vapor Deposition (LPCVD) (Levin \& Adams 1982) and Plasma Enhanced Chemical Vapor Deposition (PECVD) (Pillote et al 1993) are commonly used techniques for preparing PSG films. All the three processes commonly use silane or TEOS (for source of silicon) and phosphine (for source of phosphorus) as precursor for the chemical reaction. Silane and phosphine gases are very toxic requiring very elaborate safety measures. Also, all these deposition process are carried out at relatively high temperatures.

*For correspondence 
In the present work, we report RF magnetron sputtered PSG films as a sacrificial layer for MEMS. The RF sputtering has some advantages over CVD techniques as no toxic and pyrophoric gases $\left(\mathrm{SiH}_{4}, \mathrm{PH}_{3}\right.$, etc.) are involved in the deposition process. Also RF sputtering is a low temperature process. The right combination of the structural and sacrificial layer material and sacrificial layer etching are some of the important issues in surface micromachining technology (Ristic 1994). For fabricating large-sized free-standing structures, it is a requirement that the etch rate of the sacrificial layer be very high and the etching selectivity with respect to the structural layer should also be very large (Gad-el-Hak 2002). As reported in the literature, CVD deposited PSG films is the most suitable material for sacrificial layer because of its high etch rate. Due to the above-mentioned advantages of RF sputtering over CVD process, we have explored the RF sputtered PSG film as a sacrificial layer in surface micromachining technology. To demonstrate the process compatibility, micro-cantilevers of RF sputtered silicon dioxide/silicon nitride were fabricated using RF sputtered PSG as a sacrificial layer in surface micromachining.

\section{Experimental work}

A target of silicon dioxide mixed with phosphorus was prepared in-house for depositing the PSG films by RF sputtering process and the phosphorus concentration was estimated in the PSG films (Chandra \& Bhatt 2006). The deposition of PSG (sacrificial layer) and $\mathrm{SiO}_{2}$ (structural layer) films was carried out using RF (13.56 MHz) magnetron/diode sputtering process respectively in sputter-up configuration (Alcatel QM-311). The deposition parameters for both the films are listed in table 1. No external substrate heating was used during PSG and $\mathrm{SiO}_{2}$ film deposition. The deposition rates were obtained by measuring the film thickness using a thin-film analyzer (Filmetrics F20). The deposition rate was obtained from the measured thickness and the deposition time. The temperature of the wafer surface was measured by cementing a thermocouple on its front-surface. The etch rate of the PSG films is an important parameters for its application in surface micromachining process. High etch rates are desired especially for releasing large-size microstructures. The etch rate of PSG film was measured in buffered HF. The residual stress in PSG and $\mathrm{SiO}_{2}$ film is an important parameter when these are used for MEMS applications. This was estimated by measuring the change in the radius of curvature of the wafer, before and after deposition, using 500 TC temperature controlled film stress measurement system (FSM Frontier Semiconductor). The substrate curvature method relies on the Stoney formula (Ohring 2002) relating the average stress of the film to the

Table 1. Deposition parameters for $\mathrm{RF}$ sputtered $\mathrm{SiO}_{2}$ and PSG films.

\begin{tabular}{lll}
\hline & \multicolumn{1}{c}{$\mathrm{SiO}_{2}$ film } & \multicolumn{1}{c}{ PSG film } \\
\hline Base vacuum & $2 \times 10^{-6}$ Torr & $2 \times 10^{-6}$ Torr \\
Target & 3 -inch diameter, circular, $\mathrm{SiO}_{2}$ & 3 -inch diameter, circular, PSG \\
Sputtering gas & $\mathrm{Ar}$ & $\mathrm{Ar}$ \\
Sputtering pressure & $5 \mathrm{mTorr}$ & $20 \mathrm{mTorr}$ \\
Sputtering power & $300 \mathrm{watt}$ & $300 \mathrm{watt}$ \\
Substrate-target distance & $45 \mathrm{~mm}$ & $45 \mathrm{~mm}$ \\
Deposition rate & $120 \AA ̊ \mathrm{~min}$ & $325 \AA / \mathrm{min}$ \\
\hline
\end{tabular}


substrate curvature under the assumption that the film is much thinner than the underlying substrate. The stress $\left(\sigma_{f}\right)$ in a thin film of thickness $d_{f}$ can be expressed as

$$
\sigma_{f}=\frac{E_{s} d_{s}^{2}}{6\left(1-v_{s}\right) d_{f}}\left(\frac{1}{R_{f}}-\frac{1}{R_{0}}\right),
$$

where $E_{s}, v_{s}$ and $d_{s}$ are Young's modulus, Poisson ratio and thickness of the substrate, respectively and $R_{0}$ and $R_{f}$ are the radii of substrate curvature before and after film deposition.

The surface roughness of the PSG films before and after CMP was measured using an atomic force microscope (Nanoscope III A, Digital Instruments). MEMS structures such as microcantilever and micro-bridge were fabricated using RF sputtered silicon dioxide as a structural material and RF sputtered PSG as a sacrificial layer. A two-mask process was followed for this purpose. Figure 1 shows the schematic illustration of the process sequence. First, PSG of four-micron thickness was deposited on (100) silicon wafer by RF sputtering. The PSG is then patterned using photolithography process. After this step, the silicon dioxide of two-

\section{(100)-Si}

(a) Deposition of sacrificial layer (PSG)

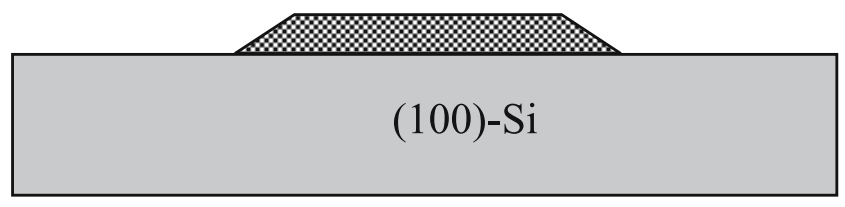

(b) Patterning of PSG

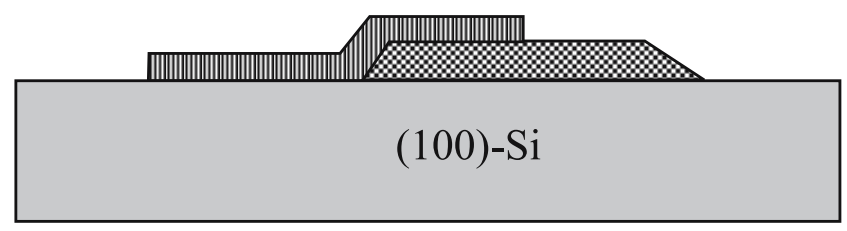

(c) Deposition and patterning of $\mathrm{SiO}_{2}$

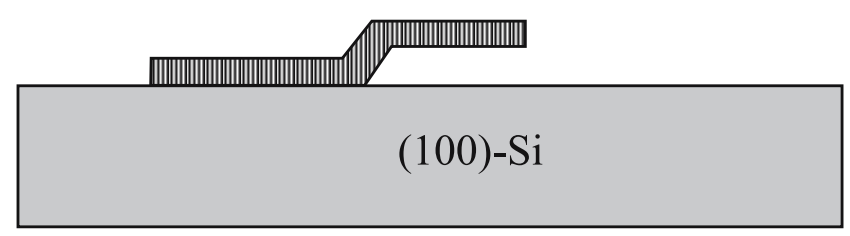

(d) Removal of PSG
Figure 1. Schematic process sequence for fabricating $\mathrm{SiO}_{2}$ micro-cantilever beam using RF sputtered PSG as a sacrificial layer by surface micromachining process. 


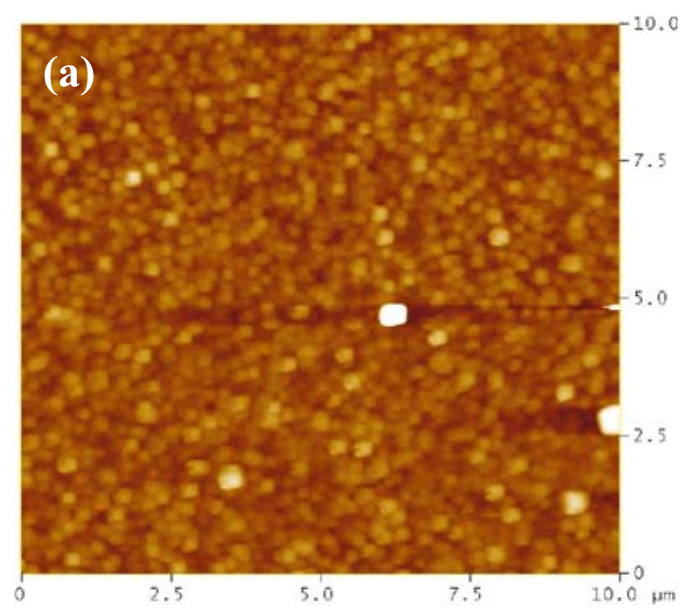

Image Statistics

\begin{tabular}{ll|}
\hline Img. Z range & $80.143 \mathrm{~nm}$ \\
Img. Mean & $0.000000 \mathrm{~nm}$ \\
Img. Raw mean & $227.50 \mathrm{~nm}$ \\
Img. Rms (Rq) & $3.793 \mathrm{~nm}$ \\
Img. Ra & $2.548 \mathrm{~nm}$ \\
Img. Rmax & $80.143 \mathrm{~nm}$ \\
\hline
\end{tabular}

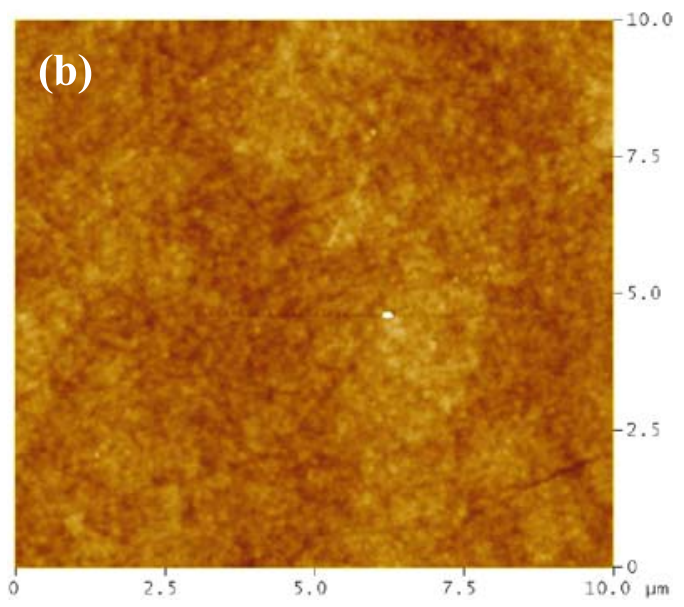

Image Statistics

\begin{tabular}{ll|} 
Img. Z range & $10.613 \mathrm{~nm}$ \\
Img. Mean & $0.000000 \mathrm{~nm}$ \\
Img. Raw mean & $45.294 \mathrm{~nm}$ \\
Img. Rms (Rq) & $0.281 \mathrm{~nm}$ \\
Img. Ra & $0.216 \mathrm{~nm}$ \\
Img. Rmax & $10.613 \mathrm{~nm}$
\end{tabular}

Figure 2. AFM image for (a) as deposited PSG film and (b) after chemical mechanical polishing (CMP).

micron thickness was deposited at $300 \mathrm{~W}$ RF power and $5 \mathrm{mTorr}$ pressure for microstructure realization. The next masking step is carried out to define the microstructures and the silicon dioxide is etched out in buffered hydrofluoric acid (BHF). Finally, the sacrificial layer is removed in BHF. It takes about 10 minutes to release the cantilevers of $20 \mu \mathrm{m}$ width and $50 \mu \mathrm{m}$ length. Following the PSG etch, the samples were rinsed in two successive DI water bath, followed by $10 \mathrm{~min}$ rinse in acetone and $10 \mathrm{~min}$ in surface tension weakening solution (methanol) and finally dried under an IR lamp.

\section{Results and discussion}

Sputtered silicon dioxide film, which is explored as a structural layer material in the present work, was deposited at 5 mTorr pressure and 300 watt power (in the RF diode configuration) and the deposition rate was measured to be $120 \AA / \mathrm{min}$. As mentioned earlier, the PSG films 

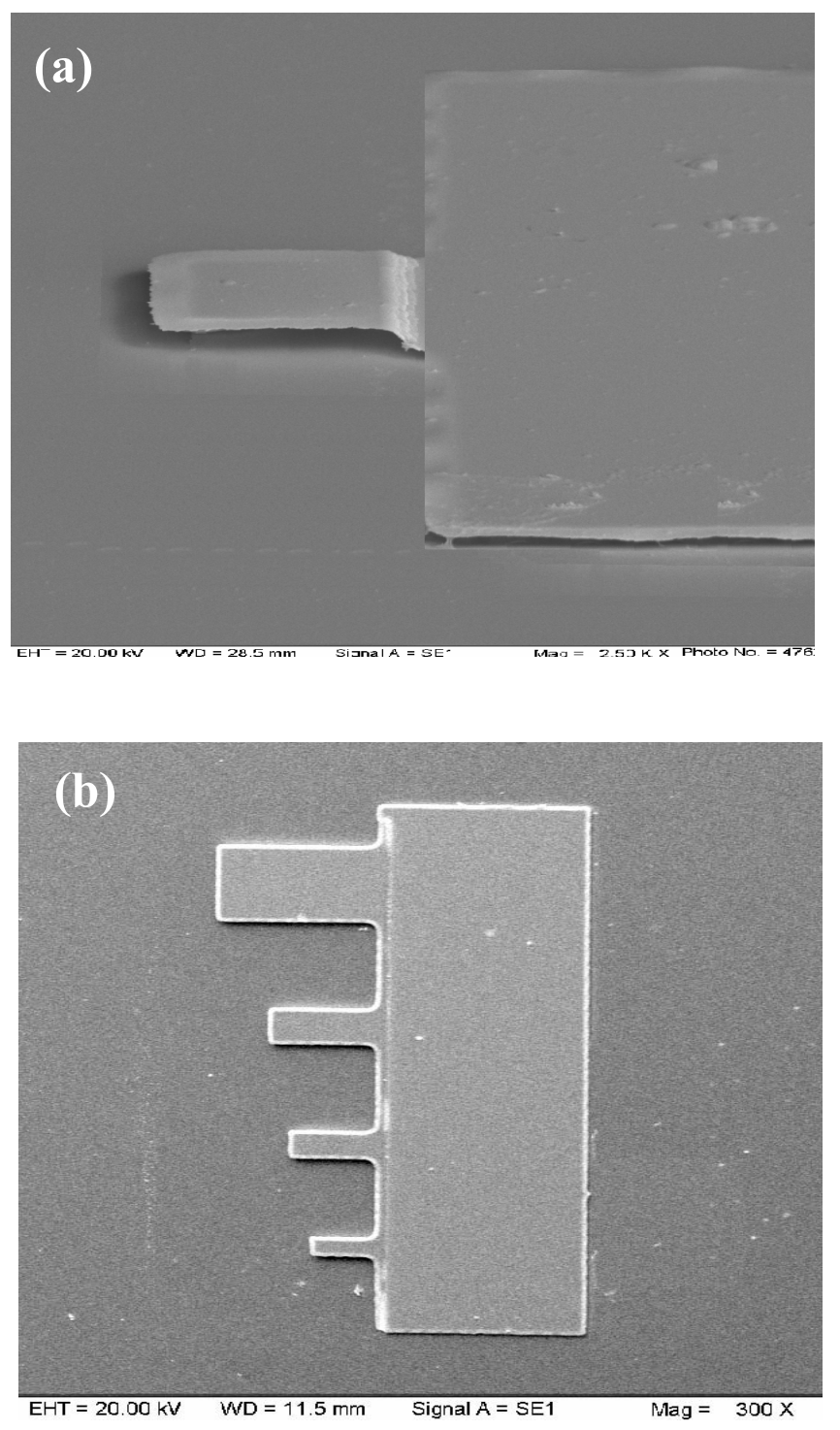

Figure 3. SEM photograph of RF sputtered $\mathrm{SiO}_{2}$ (a) microcantilever beam and (b) an array of cantilever beam using RF sputtered PSG as a sacrificial layer fabricated by surface micromachining technology.

were deposited in magnetron mode at 20 mTorr pressure, 300 watt power, and $45 \mathrm{~mm}$ separation between the target and the substrate. The deposition rate under these conditions was measured to be $325 \AA / \mathrm{min}$. The maximum temperature of the substrate during the deposition of PSG and $\mathrm{SiO}_{2}$ was measured to be $120 \pm 5^{\circ} \mathrm{C}$ and $280 \pm 5^{\circ} \mathrm{C}$ respectively for the above mentioned deposition parameters.

For releasing large-sized structures in reasonable time, sacrificial layer etch-rate is desired to be high. The PSG film deposited at 300 watt RF power and 20 mTorr pressure was found to have reasonably high etch-rate $(4 \mathrm{micron} / \mathrm{min})$ in BHF solution. This high etch rate of the RF sputtered PSG film in BHF makes this a suitable material for sacrificial layer in MEMS fabrication. PSG film deposited at 20 mTorr pressure and 300 watt RF power shows compressive stress and its value was measured to be $2 \mathrm{MPa}$. Residual stress in $\mathrm{SiO}_{2}$ films is 
of utmost importance as these films are used as a structural layer. Higher stress can create upward or downward bending in the beams. $\mathrm{SiO}_{2}$ film deposited at $5 \mathrm{mTorr}$ pressure and 300 watt RF power shows compressive stress and its value was measured to be about $90 \mathrm{MPa}$. Figures $2 \mathrm{a}$ and $\mathrm{b}$ show the AFM of PSG film before and after chemical mechanical polishing (CMP). Figure 3 shows the SEM photograph of silicon dioxide cantilever beam formed using sputtered PSG film as sacrificial layer.

\section{Conclusions}

RF magnetron sputtered PSG films have been successfully demonstrated as a sacrificial layer in surface micromachining technology. For this purpose, a target was prepared by solid-state reaction route. The etch rate of the PSG film deposited at 20 mTorr pressure and 300 watt RF power in buffered HF was found to be about 30 times higher $(\sim 4 \mu \mathrm{m} / \mathrm{min})$ as compared to that of thermal oxide. This adequately meets the requirement of sacrificial layer in surface micromachining technology.

\section{References}

Chandra S, Bhatt V 2006 Proceedings of the SPIE 6415: 64151H 1-9

Gad-el-Hak M 2002 The MEMS Handbook (USA: CRC Press)

Kern W, Schnable G L 1982 Chemically vapor-deposited borophosphosilicate glasses for silicon device applications. RCA Rev. 43: 423

Levin R M, Adams A C 1982 Low pressure deposition of phosphosilicate glass films. J. Electrochem. Soc. 129: $1588-1592$

Ohring M 2002 The material science of thin film, deposition and structure. Mechanical properties of thin films. Second ed. (New York: Academic Press)

Pillote C L, Shemansky F A, Cale T S, Raupp G B 1993 Characterization of phosphosilicate glass films obtained using plasma-enhanced chemical vapor deposition from tetraethylorthosilicate and trimethylphosphite. Thin Solid Films 236: 287-293

Ristic L 1994 Sensor Technology and Devices (MA: Artech House, Inc.)

Sze S M, 1988 VLSI Technology, (New York: McGraw-Hill Book Company)

Takamatsu A, Shibata M, Sakai H, Yoshimi T 1984 Plasma-activated deposition and properties of phosphosilicate glass film. J. Electrochem. Soc. 131: 1865-1870 